\title{
Hesitant fuzzy multi-attribute decision making based on binary connection number of set pair analysis
}

\author{
Qing Shen ${ }^{1,3} \cdot$ Jungang Lou ${ }^{2,3} \cdot$ Yong $\operatorname{Liu}^{4} \cdot$ Yunliang Jiang ${ }^{2,3}$ (1) \\ Accepted: 23 August 2021 / Published online: 4 October 2021 \\ (c) The Author(s), under exclusive licence to Springer-Verlag GmbH Germany, part of Springer Nature 2021
}

\begin{abstract}
To objectively evaluate the influence of hesitant fuzziness on the ranking of alternatives in multi-attribute decision making with hesitant fuzzy or probabilistic hesitant fuzzy information, the binary connection number of set pair analysis is applied to hesitant fuzzy multi-attribute decision making. The hesitant or probabilistic hesitant fuzzy set is transformed to the binary connection number. A hesitant fuzzy multi-attribute decision making model based on binary connection number is then established. Binary connection number theory is utilized to obtain the hesitant fuzzy center and decision-making suggestions about the alternative ranking under different hesitant fuzzy conditions. Experimental examples show that the hesitant fuzzy multi-attribute decision making model based on binary connection number has a certain versatility. It can determine the optimal scheme under the influence of hesitant fuzziness on the alternative ranking and contains the results of the same hesitant fuzzy decision-making problem using other methods, which helps in targeted decision making according to different hesitant fuzzy conditions.
\end{abstract}

Keywords Hesitant fuzzy sets · Probabilistic hesitant fuzzy sets · Multi-attribute decision making · Binary connection number $\cdot$ Conditional decision making

\section{Introduction}

Decision making is a mental activity. Due to the differences in the cognition of conditions and the complexity of decision-making problems, there is always a certain hesitation and fuzziness in qualitative judgments and quantitative characterizations of decision-making elements. Researchers have recently applied the hesitant fuzzy set (HFS)(Torra and Narukawa 2009; Torra 2010) to fuzzy decision making. HFS theory allows the membership degree of an element of a set to take several hesitant fuzzy values, which can better

Yunliang Jiang

jyl@zjhu.edu.cn

1 School of Science and Engineering, Huzhou College, Huzhou 313000, Zhejiang, China

2 School of Information Engineering, Huzhou University, Huzhou 313000, Zhejiang, China

3 Zhejiang Province Key Laboratory of Smart Management and Application of Modern Agricultural Resources, Huzhou, China

4 Institute of Cyber-Systems and Control, Zhejiang University, Hangzhou 310027, Zhejiang, China reflect the preferences and hesitation of decision makers. Its resemblance to a real decision-making environment has popularized the study of the multi-attribute decision-making (MADM) problem in hesitant fuzzy environment based on HFS theory (Ali and Rashid 2019; Farhadinia and HerreraViedma 2019; Ma et al. 2019; Liao et al. 2018; Qin et al. 2016; Xia and Xu 2011). Rodriguez et al. (2012) proposed the hesitant fuzzy linguistic term set (HFLTS), based on a fuzzy linguistic approach, to increase the flexibility and richness of linguistic elicitation, and established a multi-criteria linguistic decision-making model to manage linguistic expressions represented by HFLTS. Yang and Hussain (2019) constructed new distance and similarity measures between HFSs and applied them to multi-criterion decision making and clustering. Zhao et al. (2017) defined the hesitant fuzzy expected value and established two optimization models to gain the attribute weights in decision-making. Xia and Xu (2011) discussed the concept of the hesitant fuzzy element (HFE) to represent the degree to which an alternative satisfies an attribute, and developed some operations and aggregation operators for it. Mishra et al. (2021) introduced a novel divergence measure for hesitant fuzzy sets to identify the key 
challenges of medical decision making during the COVID-19 pandemic. Saha et al. (2021) developed some hybrid hesitant fuzzy weighted aggregation operators and presented a procedure of MADM based on the proposed operators under a hesitant fuzzy environment. Krishankumar et al. (2021a) proposed a new decision framework under a double-hierarchy hesitant fuzzy linguistic term set context for rational decision making and validated the applicability of this term set by solving the green supplier selection problem. However, the HFS assumes that the probability of different membership degrees is consistent. To effectively consider the probability that each membership degree occurs, Zhu (2014) defined a probability hesitant fuzzy set (P-HFS), which considers membership degrees and provides their probabilities, and can better describe the psychological preferences of decision makers. Krishankumar et al. (2021b) presented a new decision framework under an interval-valued probabilistic hesitant fuzzy set context with fully unknown weight information and validated the framework applied to green supplier selection for a leading bakery company. Krishankumar et al. (2021c) presented a systematic procedure for the estimation of occurrence probability of each hesitant fuzzy element, that provided sensible and rational probability hesitant fuzzy elements for evaluation. A new ranking method was also proposed that extended a well-known VIKOR method to the probability hesitant fuzzy set context. Although these algorithms express the hesitant fuzziness of decision evaluation values with HFSs or P-HFSs, the model does not objectively reflect the influence of the hesitant fuzzy intensity of decision evaluation values on the ranking of schemes. The resulting decision suggestions have difficulty reflecting the hesitant fuzziness of the decision conditions and environment, making it necessary to explore a new method of hesitant fuzzy multi-attribute decision making (HFMADM).

The binary connection number (BCN) is a mathematical concept in set pair analysis (SPA) theory, which can simultaneously represent a system's certainty and uncertainty measures, along with their relationship. It has been applied in artificial intelligence and uncertain decision making (Shen et al. 2020; Garg and Kumar 2018a; Grag and Kumar 2018b; Garg and Kumar 2019, 2020; Kumar and Garg 2018a,b). Kumar and Garg (2018a,b) proposed technology for order preference by similarity (TOPSIS) to solve decision-making problems under intuitionistic and interval-valued intuitionistic fuzzy environments using the BCN in SPA theory. Cao et al. 2018 used connection degree to represent interval-valued intuitionistic fuzzy information, and transformed intervalvalued intuitionistic stochastic decision-making matrices to connection degree matrices for interval-valued intuitionistic stochastic multi-criterion decision-making (MCDM) problems. Alternatives are ranked according to values of set pair potential. We use BCN to analyze the effect of hesitant fuzziness on the ranking of schemes in HFMADM. By converting decision-making evaluations expressed with HFSs or P-HFSs to the BCN $A+B i$, we establish the HFMADM model based on $\mathrm{BCN}$. We can use different values of $i$ in $\mathrm{BCN}$ for a preference order analysis of $m$ alternatives in a hesitant fuzzy environment. Decision-making suggestions are given in different conditions according to the rankings. The objective of the work is explained as follows.

1. The binary connection number in set pair analysis is introduced into the context of hesitant fuzzy sets to handle the complex multi-attribute decision-making problems.

2. An extended hesitant fuzzy decision-making model using the introduced binary connection numbers that can simultaneously represent the certainty and uncertainty measures of the system is established.

3. The practicality of the proposed decision model is demonstrated using two examples under hesitant fuzzy environment and probabilistic hesitant fuzzy environment. Moreover, the strengths and weaknesses of the framework are discussed by comparison with other methods.

\section{Preliminaries}

We review some basic definitions and notations.

\subsection{HFSs and HFEs}

Definition 1 (Torra 2010; Xia and $\mathrm{Xu}$ 2011) Let $V$ be a nonempty finite universe, and call $A=\left\{\left\langle x, h_{A}(x)\right\rangle \mid x \in V\right\}$ the HFS on $V$, where $h_{A}(x)$ is a set of some different values in $[0,1]$, representing the possible membership degrees of the element $x \in V$ to the set $A$. We call $h_{A}(x) \mathrm{HFE}$, and an HFS is a set of HFEs. When $h_{A}(x)$ has only one element, the HFS degenerates into a classic fuzzy set.

\subsection{P-HFSs and probabilistic hesitant fuzzy elements (P-HFEs)}

Definition 2 (Gou and $\mathrm{Xu}$ 2016) Let $X$ be a nonempty set. A P-HFS on $X$ is a mapping of a probability distribution function that, when applied to $X$, returns a subset of $[0,1]$, expressed as $H=\left\{\left\langle x, h_{x}\left(p_{x}\right)\right\rangle \mid x \in X\right\}$, where $h_{x}$ and $p_{x}$ are subsets of $[0,1] . h_{x}$ denotes the possible membership degrees of element $x \in X$ to the set $H$, and $p_{x}$ denotes the possibilities associated with $h_{x}$ satisfying $\sum p_{x}=1 . h_{x}\left(p_{x}\right)$ is called a P-HFE. For convenience, we denote the P-HFE $h_{x}\left(p_{x}\right)$ as $h(p)=\left\{\gamma_{l}\left(p_{l}\right)|l=1,2, \ldots| h,(p) \mid\right\}$, where $p_{l}$ is the probability of the possible membership degree $\gamma_{l}$, which satisfies $\sum_{l=1}^{|h(p)|} p_{l} \leq 1$, where $|h(p)|$ is the number of all membership degrees. 


\subsection{Binary connection number (BCN)}

The BCN was proposed by Zhao (2008) when he interpreted Russells paradox in set theory, also called the barber paradox, which states that there is a barber who declares that "I only cut hair for people who do not cut their own hair." According to set theory, all people who do not cut their own hair are regarded as a set $A$, so the barber belongs to $A$. The question is whether the barber cuts his own hair. According to his own rules, he should cut his own hair, i.e., he cannot belong to $A$. The barber paradox caused the third crisis in the history of mathematical development. Zhao Keqin defined another set, $B$, expressed its uncertainty, with an undetermined coefficient $i \in[-1,1]$, and combined $A$ and $B$ into a set pair $H=(A, B)$, consisting of all objects the barber should serve. For the sake of brevity, $A+B i$ is directly used as the feature function of $H$, where $i=1$ when the barber cuts his own hair, and otherwise $i=-1$. This avoids the paradox, and the problem is transformed to the value analysis of $i$ in $A+B i$, which is called a BCN. We use BCNs to research HFMADM, defining a $\mathrm{BCN}$ as follows.

Definition 3 Assume that two sets $E$ and $F$ are needed for the objective description of an object, where $E$ is a deterministic set, and the elements $e_{1}, e_{2}, \ldots, e_{n}$ are determined to belong to $E$; and $F$ is a hesitant fuzzy set, whose elements $f_{1}, f_{2}, \ldots, f_{m}$ can each have more than one membership degree $v(v \in(0,1))$, not equal to 1 , of belonging to $F$. Then the system formed by the sets $E$ and $F$ is called a hesitant fuzzy set pair. If $H$ is a hesitant fuzzy set pair, then

$H=(E, F)$.

The hesitant fuzzy set pair is called a set pair for convenience.

Definition 4 Let $H=(E, F)$ be a hesitant fuzzy set pair, with hesitant fuzzy center $A$ and hesitant fuzzy radius $B$, and let $i(i \in[-1,1])$ denote the hesitant fuzzy direction and the hesitant fuzzy intensity of $B(F)$. Then, $\mathrm{A}+\mathrm{Bi}$ is the characteristic function of the hesitant fuzzy set pair $H=(E, F)$, which is called the binary hesitant fuzzy connection number, and $i$ is called the hesitant fuzzy intensity function. The interval $[-1,1]$ formed by the maximum hesitation fuzzy strength $(i=1)$ and minimum hesitant fuzzy strength $(i=-1)$ is called the hesitation fuzzy region. Let $u$ represent a binary hesitant fuzzy connection number. Then

$u=A+B i$.

Definition 5 Let $A+B i$ be the characteristic function of the hesitant fuzzy set pair $H=(E, F)$, and let $A+B=N$. Then $N$ is called the hesitant fuzzy connection norm. Divide both sides of Eq. (2) by $N$, and let $\mu=\frac{u}{N}, a=\frac{A}{N}, b=\frac{B}{N}$.
Then

$\mu=a+b i$

Eq. (3) is called the normalized binary hesitant fuzzy connection number, with a constraint,

$a+b=1$,

unlike Eq. (2). The normalization binary hesitant fuzzy connection number is expressed as

$\mu=a+b i\left\{\begin{array}{l}a \in[0,1] \\ b \in[0,1] \\ a+b=1 \\ i \in[-1,1]\end{array}\right.$,

and is also referred to as the $\mathrm{BCN}$ or connection number $(\mathrm{CN})$.

\subsubsection{Properties of $B C N$}

\section{Systematicness}

According to Definition 1, the object described by the $\mathrm{BCN}$ $a+b i$ is a system; mathematical expressions about the objects certainty measure $a$, uncertainty measure $b$, and their correlations in the $\mathrm{BCN}$ form a system; and the relation between $a$ and $b$ has two levels. The sum relationship $a+b$ is at the currently visible macroscopic level, and the value of $i$ must be further analyzed at the microscopic level. This indicates that the $\mathrm{BCN} a+b i$ is a hierarchical system.

\section{Hesitant fuzziness}

The value of $i$ in the BCN must be analyzed at the microscopic level, which shows the hesitation compared with the currently given $a+b=1$. While $i$ takes specific values at different levels, the hesitancy objectively leads the value of the $\mathrm{BCN}$ to have hesitant fuzziness.

\section{Comparability}

Case 1 According to Eq. (5) in Definition 5, it is easy to know that $\left(a+\left.b i\right|_{i=1}\right)>\left(a+\left.b i\right|_{i=0}\right)>\left(a+\left.b i\right|_{i=-1}\right)$.

Case $2 \mathrm{It}$ is further inferred from case 1 that when there are certain values of $i_{1}$ and $i_{2}$ in BCNs $\mu_{1}$ and $\mu_{2}$, respectively, these $\mathrm{BCNs}$ can be compared.

For example, if $\mu_{1}=0.6+0.4 i_{1}$ and $\mu_{2}=0.5+0.5 i_{2}$, then when $i_{1}=0.3, i_{2}=0.5, \mu_{1}<\mu_{2}$; and when $i_{1}=0.5$, $i_{2}=0.3, \mu_{1}>\mu_{2}$. 


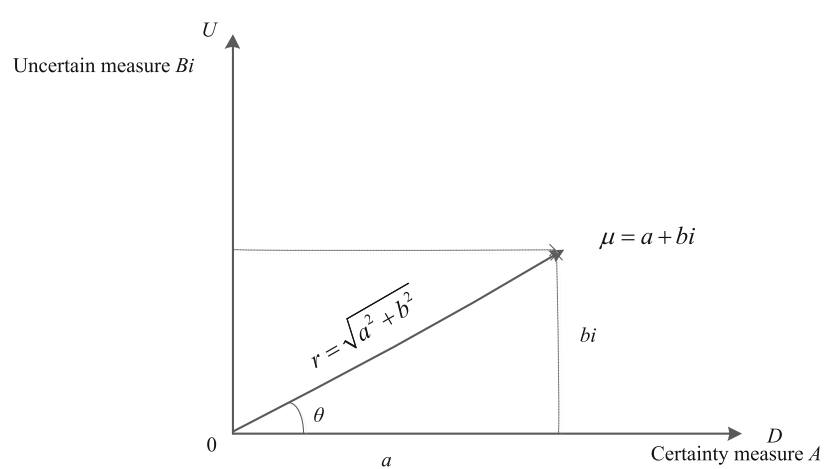

Fig. 1 Module of binary connection number $\mu=a+b i$ in D-U space

Case 3 the $\mathrm{BCN} \mu_{n}=a_{n}+b_{n} i$ can be mapped to the D-U space, as shown in Fig. 1. The modulus of the BCN can be calculated by

$r_{n}=\sqrt{a_{n}^{2}+b_{n}^{2}}$

According to the magnitude relationship of the modulus $r_{n}$, the magnitude relationship of $n$ BCNs $\mu_{n}=a_{n}+b_{n} i$ is determined. The principle is to treat the $\mathrm{BCN}$ as a pair of vectors, and to find their combination in D-U space.

It can be seen from Fig. 1 that the modulus of the BCN $a+b i$ is a combination of the certainty measure $a$ and the uncertainty measure $b i$ when $i=1$.

\subsection{Trigonometric representation of $\mathrm{BCNs}$}

Using the relations $a=r \cos \theta$ and $a=r \sin \theta$ of the rectangular coordinate system, the trigonometric function formula of the BCN $a+b i$ is expressed as (Zhao 2008)

$\mu=r(\cos \theta+i \sin \theta)$,

where $\theta=\arctan \frac{b}{a}$ is called the angle of the $\mathrm{BCN}$.

\subsection{Operations of BCNs}

BCNs can be used for the ordinary operations of addition, subtraction, multiplication, and division, but we use only addition and multiplication, which are defined as follows.

Definition 6 Let $\mu_{1}=a_{1}+b_{1} i, \mu_{2}=a_{2}+b_{2} i$ be two BCNs. Then their sum is a $\mathrm{BCN} \mu=a+b i$, denoted as

$\mu=\mu_{1}+\mu_{2}$,

where $a=a_{1}+a_{2}, b=b_{1}+b_{2}$. It can be seen that the addition of BCNs satisfies the commutative law, and the addition of three or more BCNs satisfies the associative law.
Definition 7 Let $\mu_{1}=a_{1}+b_{1} i, \mu_{2}=a_{2}+b_{2} i$ be two BCNs. Then, their product is a $\mathrm{BCN}, \mu=a+b i$, denoted as

$\mu=\mu_{1} \times \mu_{2}$,

where $\mu_{1} \times \mu_{2}=\left(a_{1}+b_{1} i\right) \times\left(a_{2}+b_{2} i\right)=a_{1} a_{2}+a_{1} b_{2} i+$ $a_{2} b_{1} i+b_{1} b_{2} i^{2}$. When we only consider the relationship between certainty and uncertainty, regardless of the level (power) of uncertainty, we can make $i^{n}=i$, so $a=a_{1} a_{2}$, $b=a_{1} b_{2}+a_{2} b_{1}+b_{1} b_{2}$.

Definition 8 If a nonezero real number $k(k \neq 0)$ is multiplied by a $\mathrm{BCN} \mu=a+b i$, the product is still a $\mathrm{BCN}$,

$k \mu=k(a+b i)=k a+k b i$.

The pairwise principle of SPA states that things or concepts always exist in pairs. Hence, BCNs have both scalar and vector properties. According to the scalar property, we can define the algebraic operation of BCNs by the polynomial operation rules of ordinary algebra; according to the vector property, we can define the synthesis operation of two connection components in $\mathrm{BCNs}$ by the vector operation rules of complex numbers. When solving practical problems, an operation rule can be selected as required, or two kinds of operations can be used at the same time, and results obtained by mutual conversion and complementarity in the operation process can be used to solve the problem completely.

\section{Transformation from HFSs or P-HFSs to BCNs}

\subsection{Transformation from HFSs to $B C N$}

Definition 9 Let $X$ be a fixed set. An HFS on $X$ is $A=$ $\left\{\left\langle x, h_{A}(x)\right\rangle \mid x \in X\right\}$, with HFEs $h_{A}(x)=\left(x_{1}, x_{2}, \ldots, x_{n}\right)$, and let

$a=\overline{h_{A}(x)}=\frac{\sum x_{n}}{n}$.

Then $a$ is called the average hesitant fuzzy degree, or the hesitant fuzzy expectation or hesitant fuzzy center of the HFEs $h_{A}(x)=\left(x_{1}, x_{2}, \ldots, x_{n}\right)$. Let

$b=\max \left(x_{1}, x_{2}, \ldots, x_{n}\right)-\overline{h_{A}(x)}$.

Then $b$ is called the distance between the hesitant fuzzy boundary and the hesitant fuzzy center of the HFEs $h_{A}(x)=$ $\left(x_{1}, x_{2}, \ldots, x_{n}\right)$ and is also called the hesitant fuzzy radius. Equations (11) and (12) are called the conversion formula from the HFEs $h_{A}(x)=\left(x_{1}, x_{2}, \ldots, x_{n}\right)$ to the BCN. Equation (11) extracts the relative certainty in the HFEs 
$h_{A}(x)=\left(x_{1}, x_{2}, \ldots, x_{n}\right)$, which is a relatively certain hesitant fuzzy measure; Eq. (12) shows the relative uncertainty in the HFEs $h_{A}(x)=\left(x_{1}, x_{2}, \ldots, x_{n}\right)$, which is a relative uncertain hesitant fuzzy measure. In the $\mathrm{BCN}$, the relation between the relative certain hesitant fuzzy measure and the relative uncertain hesitant fuzzy measure in the HFEs $h_{A}(x)=\left(x_{1}, x_{2}, \ldots, x_{n}\right)$ is expressed by algebraic addition.

From the perspective of probability theory, the above conversion is equivalent to treating an $\operatorname{HFE} h_{A}(x)=$ $\left(x_{1}, x_{2}, \ldots, x_{n}\right)$ as sample data, taking the average $\overline{x_{n}}$ and deviation $s$ of $x_{1}, x_{2}, \ldots, x_{n}$ as the parameters reflecting the HFE as a whole. Therefore, when $n$ of $h_{A}(x)=$ $\left(x_{1}, x_{2}, \ldots, x_{n}\right)$ is large, e.g., $n \geq 10$, the variance of $h_{A}(x)=\left(x_{1}, x_{2}, \ldots, x_{n}\right)$ can be used as $b$ in the BCN to maximize the use of the hesitant fuzzy information of the HFE $h_{A}(x)$; when $n<10$, the variance can be replaced by the deviation to simplify calculations.

\subsection{Transformation from P-HFSs to BCN}

Definition 10 For a nonempty set $X=\left\{x_{t} \mid t=1,2, \ldots, n\right\}$, let P-HFS be $A_{p}=H_{p}\left\{\left\langle x_{t}, h_{A t}(p)\right\rangle \mid x_{t} \in X\right\}, h_{A t}(p)=$ $\left\{\gamma_{A t}^{l}\left(p_{A t}^{l}\right)|l=1,2, \ldots| h,(p) \mid\right\}$, and let:

$a=E\left(A_{p}\right)=\frac{1}{n} \sum_{t=1}^{n}\left(\sum_{l=1}^{|h(p)|} p_{A t}^{l} \gamma_{A t}^{l}\right)$.

Then $a$ is called the average probabilistic hesitant fuzzy degree, or the probabilistic hesitant fuzzy center. Let

$b=\operatorname{Var}\left(A_{p}\right)=\frac{1}{n} \sum_{t=1}^{n}\left(\sum_{l=1}^{|h(p)|} p_{A t}^{l} \gamma_{A t}^{l}-E\left(A_{p}\right)\right)^{2}$.

Then $b$ is called the probabilistic hesitant fuzzy radius. Equations (13) and (14) are called the conversion formulas from P-HFEs to BCN.

Thus, the P-HFS can be transformed to the $\mathrm{BCN}$, i.e.,

$$
\begin{aligned}
\mu= & a+b i=\frac{1}{n} \sum_{t=1}^{n}\left(\sum_{l=1}^{|h(p)|} p_{A t}^{l} \gamma_{A t}^{l}\right) \\
& +\frac{1}{n} \sum_{t=1}^{n}\left(\sum_{l=1}^{|h(p)|} p_{A t}^{l} \gamma_{A t}^{l}-E\left(A_{p}\right)\right)^{2} i .
\end{aligned}
$$

\section{HFMADM problems}

\subsection{Problem statement}

Suppose there is a discrete set of $m$ optional schemes, $S=\left\{S_{1}, S_{2}, \ldots, S_{m}\right\}$. Let $X$ be the discussion universe containing the attributes, where each scheme has $n$ attributes, and $X=\left\{x_{1}, x_{2}, \ldots, x_{n}\right\}$ is the set of all attributes. Experts provide the evaluation values $P_{k t}$ expressed by the HFE $h_{S_{k}}\left(x_{t}\right)=\left\{\gamma \mid \gamma \in h_{S_{k}}\left(x_{t}\right), 0 \leq \gamma \leq 1\right\}$, which indicates the possible membership degrees of the $k$ th alternative $S_{k}$ under the $t$ th attribute $x_{t}$, or the P-HFE $h_{k t}\left(p_{k t}\right)=$ $\left\{\gamma_{k t}^{l}\left(p_{k t}^{l}\right)|l=1,2, \ldots| h,(p) \mid\right\}(k=1,2, \ldots, m, t=$ $1,2, \ldots, n)$ for each attribute is different; the attribute weight is $w=\left(w_{1}, w_{2}, \ldots, w_{n}\right)^{T}$, where $w_{t} \in[0,1], \sum_{t=1}^{n} w_{t}=$ 1. It is agreed that each attribute is a benefit type such that the bigger, the better. The problem is to determine the optimal among $m$ optional schemes, ranking these schemes, and performing a hesitant fuzzy analysis.

\subsection{Decision-making process}

We develop a practical approach to solve HFMADM problems, whose schematic diagram is shown as Fig. 2. We utilize an approach to obtain the preference order of all schemes, which has two cases.

Case I: Assume that the experts provide their evaluation values with HFEs; we obtain the most desirable alternative(s) according to the following steps:

Step 1 Convert the mathematical expressions of attribute values. Utilize Eqs. (11) and (12) to convert the evaluation values expressed with the HFEs $h_{p}\left(x_{k t}\right)=$ $\left(x_{k t 1}, x_{k t 2}, \ldots, x_{k t n}\right)$ given by the decision makers to BCNs,

$$
\begin{aligned}
\mu_{k t}=a_{k t} & +b_{k t} i=\frac{\sum x_{k t}}{n} \\
& +\left(\max \left(x_{k t 1}, x_{k t 2}, \ldots, x_{k t n}\right)-\frac{\sum x_{k t}}{n}\right) i .
\end{aligned}
$$

Step 2 Construct the weighted BCNs for each attribute value, i.e., the BCNs $\mu_{k t}=a_{k t}+b_{k t} i$ of each attribute value multiplied by the attribute weight $w_{t}$,

$P_{k t}=w_{t} \mu_{k t}=w_{t}\left(a_{k t}+b_{k t} i\right)=w_{t} a_{k t}+w_{t} b_{k t} i$.

Step 3 Determine the comprehensive evaluation value of each scheme. Sum $P_{k t}$ of each scheme to get its comprehensive evaluation $\mathrm{BCN}$,

$M\left(S_{k}\right)=\sum_{t=1}^{n} P_{k t}=A_{k}+B_{k} i_{k}$ 

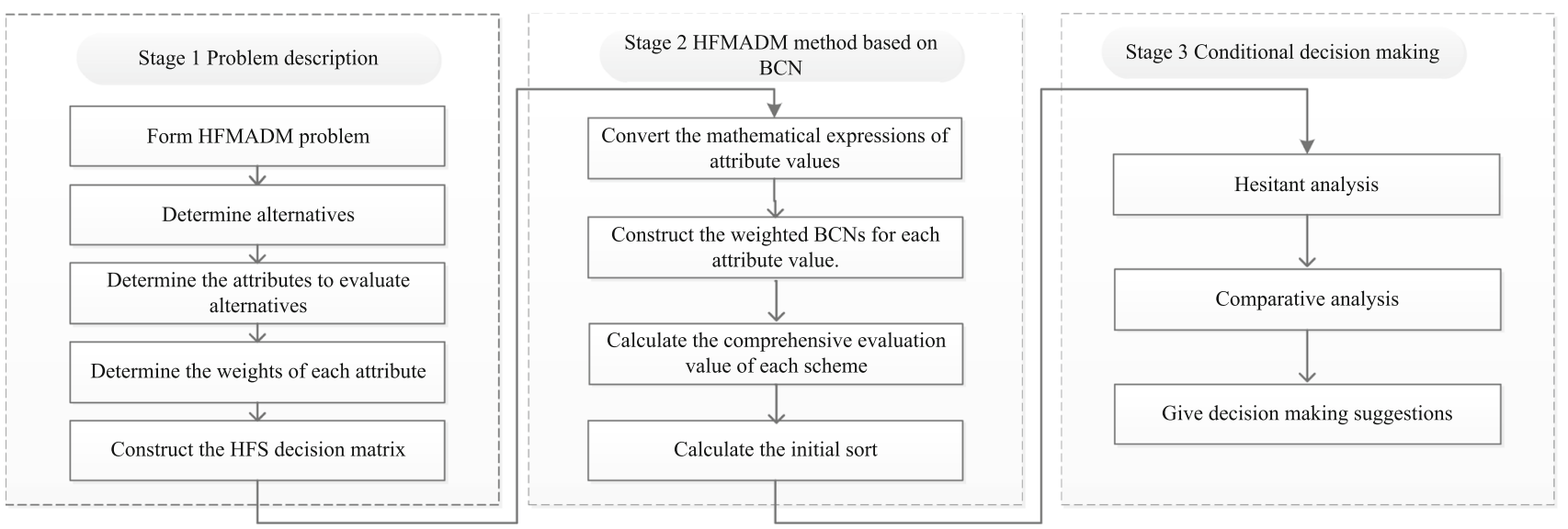

Fig. 2 Schematic diagram of proposed approach for HFMADM

Step 4 Calculate the preliminary sort. The initial ranking of $m$ schemes is only based on the value of the hesitant fuzzy center $A_{k}$. A scheme with a larger $A_{k}$ is better than one with a smaller $A_{k}$.

Step 5 Hesitant analysis. This paper discussed the influence of hesitation on the preliminary priority by using $i_{k}$ in $B_{k} i_{k}$ to take different values, and determined the most optimistic ranking $\left(i_{k}=1\right)$, the most pessimistic ranking $\left(i_{k}=-1\right)$, the sad and happy neutral ranking $\left(i_{k}=0\right)$ of all attributes, and the ranking under the condition of partial attributes being the most optimistic, partial attributes being the most pessimistic and partial attributes being the sad and happy neutral, in addition to the sorting of $m$ schemes under the condition that some attributes are optimistic $\left(i_{k} \in[0,1]\right)$ and some attributes are pessimistic $\left(i_{k} \in[-1,0]\right)$.

Step 6 Comparative analysis and decision-making suggestions. Compare the results with those of the same HFMADM problem processed by other methods to find similarities, differences, and their causes. Based on the above analysis, a decision-making method is proposed, and it is explained under which hesitant fuzzy conditions which scheme is the best and the order of other schemes.

Case II: Assume that the experts provide their evaluation values with P-HFEs; we get the most desirable alternative(s) according to the following steps. Step 1 Utilize Eqs. (13)(15) to calculate the comprehensive evaluation value of each scheme expressed by BCNs,

$$
\begin{aligned}
& M\left(S_{k}\right)=A_{k}+B_{k} i=\frac{1}{n} \sum_{t=1}^{n}\left(w_{t} \sum_{l=1}^{|h(p)|} p_{k t}^{l} \gamma_{k t}^{l}\right) \\
& +\frac{1}{n} \sum_{t=1}^{n}\left(w_{t} \sum_{l=1}^{|h(p)|} p_{k t}^{l} \gamma_{k t}^{l}-E\left(A_{p}\right)\right)^{2} i,
\end{aligned}
$$

where $E\left(A_{p}\right)=\frac{1}{n} \sum_{t=1}^{n}\left(\sum_{l=1}^{|h(p)|} p_{k t}^{l} \gamma_{k t}^{l}\right)$.
Step 2 Preliminarily rank schemes $S_{k}(k=1,2, \ldots, m)$ according to the value of the hesitant fuzzy center $A_{k}$ in step 1 , to obtain the initial sort.

Step 3 Hesitation analysis. Take different values of $i_{k}$ in the comprehensive evaluation $\mathrm{BCN}$ to discuss the effect of hesitation on the initial ranking.

Step 4 Give decision-making suggestions.

\subsection{A practical case study}

We use two practical project-selection problems to illustrate the implementation of the presented algorithm. A comparative analysis of the computational results shows its effectiveness. Finally, we propose the concept of conditional decision making.

Example 4.1 (Xia and Xu 2011) An enterprise plans to formulate a strategic investment plan within five years, to select investment projects from four possible investment project sets $S=\left(S_{1}, S_{2}, S_{3}, S_{4}\right)$. These sets are evaluated by five members of the board of directors, considering four attributes suggested by the balanced scorecard methodology (Kaplan and Norton 1996) (all of the maximization type): $v_{1}$ is financial perspective, $v_{2}$ is the customer satisfaction, $v_{3}$ is internal business process perspective, $v_{4}$ is learning and growth perspective. The weight vector of the attributes is $w=(0.2,0.3,0.15,0.35)^{T}$. The decision makers are to anonymously provide an opinion on each project. The decision matrix $H=\left(h_{k t}\right)_{m \times n}$ of the four alternatives on each attribute is shown in Table 1 , where $h_{k t}(k, t=1,2,3,4)$ are in terms of HFEs. We try to determine the optimal scheme and the preference order of the other schemes, discuss the effect of hesitation on scheme ranking, and give decision-making suggestions.

We utilize our approach to get the most desirable alternative(s) according to the following steps: 
Table 1 Hesitant fuzzy decision-making matrix

\begin{tabular}{lllll}
\hline & $v_{1}$ & $v_{2}$ & $v_{3}$ & $v_{4}$ \\
\hline$w_{t}$ & 0.2 & 0.3 & 0.15 & 0.35 \\
$S_{1}$ & $\{0.2,0.4,0.7\}$ & $\{0.2,0.6,0.8\}$ & $\{0.2,0.3,0.6,0.7,0.9\}$ & $\{0.3,0.4,0.5,0.7,0.8\}$ \\
$S_{2}$ & $\{0.2,0.4,0.7,0.9\}$ & $\{0.1,0.2,0.4,0.5\}$ & $\{0.3,0.4,0.6,0.9\}$ & $\{0.5,0.6,0.8,0.9\}$ \\
$S_{3}$ & $\{0.3,0.5,0.6,0.7\}$ & $\{0.2,0.4,0.5,0.6\}$ & $\{0.3,0.5,0.7,0.8\}$ & $\{0.2,0.5,0.6,0.7\}$ \\
$S_{4}$ & $\{0.3,0.5,0.6\}$ & $\{0.2,0.4\}$ & $\{0.5,0.6,0.7\}$ & $\{0.8,0.9\}$ \\
\hline
\end{tabular}

Table 2 BCN decision-making matrix

\begin{tabular}{lllll}
\hline & $v_{1}$ & $v_{2}$ & $v_{3}$ & $v_{4}$ \\
\hline$w_{t}$ & 0.2 & 0.3 & 0.15 & 0.35 \\
$S_{1}$ & $0.4333+0.2667 i$ & $0.5333+0.2667 i$ & $0.5400+0.3600 i$ & $0.5400+0.2600 i$ \\
$S_{2}$ & $0.5500+0.3500 i$ & $0.3000+0.2000 i$ & $0.5500+0.3500 i$ & $0.7000+0.2000 i$ \\
$S_{3}$ & $0.5250+0.1750 i$ & $0.4250+0.1750 i$ & $0.5750+0.2250 i$ & $0.5000+0.2000 i$ \\
$S_{4}$ & $0.4667+0.1333 i$ & $0.3000+0.1000 i$ & $0.6000+0.1000 i$ & $0.8500+0.0500 i$ \\
\hline
\end{tabular}

Table 3 BCN decision-making matrix with attribute weight

\begin{tabular}{lllll}
\hline & $v_{1}$ & $v_{2}$ & $v_{3}$ & $v_{4}$ \\
\hline$S_{1}$ & $0.0867+0.0533 i$ & $0.1600+0.0800 i$ & $0.0810+0.0540 i$ & $0.1890+0.0910 i$ \\
$S_{2}$ & $0.1100+0.0700 i$ & $0.0900+0.0600 i$ & $0.0825+0.0525 i$ & $0.2450+0.0700 i$ \\
$S_{3}$ & $0.1050+0.0350 i$ & $0.1275+0.0525 i$ & $0.0863+0.0338 i$ & $0.1750+0.0700 i$ \\
$S_{4}$ & $0.0933+0.0267 i$ & $0.0900+0.0300 i$ & $0.0900+0.0150 i$ & $0.2975+0.0175 i$ \\
\hline
\end{tabular}

Table 4 Comprehensive evaluation BCN for each scheme

\begin{tabular}{ll}
\hline & $M\left(S_{k}\right)=\sum_{t=1}^{4} w_{t}\left(a_{k t}+b_{k t} i_{k t}\right)=A_{k}+B_{k} i_{k}$ \\
\hline$S_{1}$ & $M\left(S_{1}\right)=0.5167+0.2783 i_{1}$ \\
$S_{2}$ & $M\left(S_{2}\right)=0.5275+0.2525 i_{2}$ \\
$S_{3}$ & $M\left(S_{3}\right)=0.4938+0.1913 i_{3}$ \\
$S_{4}$ & $M\left(S_{4}\right)=0.5708+0.0892 i_{4}$ \\
\hline
\end{tabular}

Table 5 Ranking of comprehensive evaluation $\mathrm{BCN} A_{k}+B_{k} i_{k}$ in hesitant fuzzy center $\left(i_{k}=0\right)$

\begin{tabular}{lllll}
\hline & $A_{k}\left(i_{k}=0\right)$ & Sort & $\begin{array}{l}\text { Sort using } \\
\text { Xia and Xu's } \\
\text { method }\end{array}$ & $\begin{array}{l}\text { Compared with } \\
\text { results of Xia } \\
\text { and Xu (2011) }\end{array}$ \\
\hline$S_{1}$ & 0.5167 & (3) & (3) & The same \\
$S_{2}$ & 0.5275 & (2) & (2) & The same \\
$S_{3}$ & 0.4938 & (4) & (4) & The same \\
$S_{4}$ & 0.5708 & (1) & (1) & The same \\
\hline
\end{tabular}

Step 1. Utilize Eqs. (11), (12), and (16) to convert the HFE decision matrix in Table 1 to the $\mathrm{BCN} \mu_{k t}$; the result is shown in Table 2.

Step 2. Utilize Eq. (17) to construct the weighted BCNs $w_{t} \mu_{k t}$ for each attribute value in Table 2; the result is shown in Table 3.
Table 6 Values of modulus of BCN with weight

\begin{tabular}{lllll}
\hline & $v_{1}$ & $v_{2}$ & $v_{3}$ & $v_{4}$ \\
\hline$S_{1}$ & 0.1018 & 0.1789 & 0.0974 & 0.2098 \\
$S_{2}$ & 0.1304 & 0.1082 & 0.0978 & 0.2548 \\
$S_{3}$ & 0.1107 & 0.1379 & 0.0926 & 0.1885 \\
$S_{4}$ & 0.0971 & 0.0949 & 0.0912 & 0.2980 \\
\hline
\end{tabular}

Step 3. According to the weighted BCNs in Table 3, utilize Eq. (18) to calculate the comprehensive evaluation BCN $M\left(S_{k}\right)$ for each scheme, as shown in Table 4.

Step 4 . The initial preference order of each scheme based on the $A_{k}$ value (hesitant fuzzy center) of the comprehensive evaluation BCN in Table 4 is listed in Table 5.

Step 5. Use $i_{k}$ in BCNs for hesitant fuzzy analysis.

(1) Calculate the modulus of $\mathrm{BCN}$ for each alternative in Table 2, and multiply the modulus by the attribute weight; the results are listed in Table 6.

According to Table 6, the comprehensive evaluation value of each scheme can be obtained, whose preference order is $S_{2} \succ S_{1} \succ S_{4} \succ S_{3}$.

(2) Calculate the ranking of four alternatives when $i_{k}=$ $-1,0,1$ in the comprehensive evaluation $\mathrm{BCN}$ of each alternative in Table 4; the results are listed in Table 7. 
Table 7 Ranking of schemes when $i_{k}=-1,0,1$, with HFE evaluation

\begin{tabular}{lllllll}
\hline$M\left(S_{k}\right)$ & $i_{k}=-1$ & Ranking & $i_{k}=0$ & Ranking & $i_{k}=1$ & Ranking \\
\hline$M\left(S_{1}\right)$ & 0.2383 & $(4)$ & 0.5167 & (3) & 0.7950 & (1) \\
$M\left(S_{2}\right)$ & 0.2750 & (3) & 0.5275 & $(2)$ & 0.7800 & (2) \\
$M\left(S_{3}\right)$ & 0.3025 & $(2)$ & 0.4938 & (4) & 0.6850 & (3) \\
$M\left(S_{4}\right)$ & 0.4817 & (1) & 0.5708 & (1) & 0.6600 & (4) \\
\hline
\end{tabular}

Table 8 Evaluations of four hospitals with P-HFEs

\begin{tabular}{llll}
\hline & $c_{1}$ & $c_{2}$ & $c_{3}$ \\
\hline$h_{1}$ & $\{0.5(0.4), 0.7(0.6)\}$ & $\{0.9(1)\}$ & $\{0.3(0.2), 0.5(0.8)\}$ \\
$h_{2}$ & $\{0.8(0.3), 0.9(0.7)\}$ & $\{0.5(1)\}$ & $\{0.8(0.4), 0.9(0.6)\}$ \\
$h_{3}$ & $\{0.5(1)\}$ & $\{0.7(0.5), 0.9(0.5)\}$ & $\{0.8(0.6), 0.9(0.4)\}$ \\
$h_{4}$ & $\{0.8(0.5), 0.9(0.5)\}$ & $\{0.3(0.5), 0.6(0.5)\}$ & $\{0.7(1)\}$ \\
\hline
\end{tabular}

(3) Table 7 shows that when $i_{k}=-1$, scheme $S_{4}$ is optimal, scheme $S_{3}$ is suboptimal, and the preference order of the four schemes is $S_{4} \succ S_{3} \succ S_{2} \succ S_{1}$.

When $i_{k}=0$, the ranking is that of the hesitant centers of comprehensive evaluation BCNs in Table 5; when $i_{k}=1$, scheme $S_{1}$ is optimal, and the preference order of the four schemes is $S_{1} \succ S_{2} \succ S_{3} \succ S_{4}$.

The above is an analysis of the ranking changes based on the "synchronized value" of $i$ in the comprehensive evaluation BCNs for four schemes. From the perspective of hesitant fuzziness, we should also consider the "asynchronous value" of $i$, i.e., the hesitant fuzziness of the comprehensive evaluation $\mathrm{BCN}$, including the combination of taking optimistic or pessimistic values or not sad not happy, or different fuzzy states. For example, when $i_{k}=1$ in the comprehensive evaluation BCN of scheme $S_{1}, i_{k}=0$, in the comprehensive evaluation BCNs of the scheme $S_{2}$ and $S_{3}, i_{k}=-1$ in the comprehensive evaluation BCN of the scheme $S_{4}$, the comprehensive evaluation BCNs of $S_{k}(k=1,2,3,4)$ can be obtained as $0.7950,0.5275,0.4938$ and 0.4817 , respectively. In this case, the four schemes can be ranked as $S_{1} \succ S_{2} \succ S_{3} \succ S_{4}$. When $i_{k}=1$ for scheme $S_{3}$, $i_{k}=-1$ for the other three schemes, and the relation among the corresponding comprehensive evaluation BCNs is $0.6850>0.4817>0.2750>0.2383$. In this case, the preference ranking of the four schemes is $S_{3} \succ S_{4} \succ S_{2} \succ S_{1}$, and scheme $S_{3}$ becomes the optimal alternative.

Step 6. Provide decision recommendations. Based on the above hesitant fuzziness analysis, it is found that all four alternatives are likely to be optimal in a certain hesitant fuzzy environment. In actual decision making, if only one can be implemented, the decision maker should be allowed to further consider the objective conditions for decision-making implementation, which is based on optimistic hesitant fuzzy decision selection (only considering $i_{k}=1$, equivalent to taking the maximum value $\max \left(x_{k t 1}, x_{k t 2}, \ldots, x_{k t n}\right)$ for each HFE $\left.h_{p}\left(x_{k t}\right)=\left(x_{k t 1}, x_{k t 2}, \ldots, x_{k t n}\right)\right)$, or based on the pessimistic fuzzy hesitant decision making (only considering $i_{k}=-1$, equivalent to taking the minimum value $\min \left(x_{k t 1}, x_{k t 2}, \ldots, x_{k t n}\right)$ for each $\mathrm{HFE} h_{p}\left(x_{k t}\right)=$ $\left.\left(x_{k t 1}, x_{k t 2}, \ldots, x_{k t n}\right)\right)$, or based on neutral hesitant fuzzy decision making (only considering $i_{k}=0$, equivalent to taking an average value $\bar{x}_{k t}=\frac{1}{n} \sum_{t=1}^{n} x_{k t n}$ for each HFE), or on the basis of the average value, taking into account the role of the hesitant fuzzy intensity function appropriately, which requires the decision maker to make a choice after careful consideration. If the decision maker does not want to make a decision in the case of extreme values (including the average value) of each HFE, we recommend a ranking based on the BCN "modulus $+i_{k}$ proportional value method," that is, the scheme $S_{2}$ is the optimal one, scheme $S_{1}$ is the standby one, and the preference order of four schemes is $S_{2} \succ S_{1} \succ S_{4} \succ S_{3}$. The reasons are as follows. On the one hand, the "module" of BCN is a geometric combination of "hesitant fuzzy relative certainty measure (hesitant fuzzy center)" and "hesitant fuzzy relative uncertainty measure (optimistic hesitant fuzzy)," which objectively considers the relative certainty, relative uncertainty, and their interaction in the hesitant fuzziness of the original evaluation data. On the other hand, according to the system property of the $\mathrm{BCN}$, it is known that $A_{k}$ and $B_{k}$ in $M\left(S_{k}\right)=A_{k}+B_{k} i_{k}$ are in a system, and the value of $i_{k}$ depends on the proportion of $B_{k}$ in this system, i.e., there exists the relationship

$i_{k}=\frac{B_{k}}{A_{k}+B_{k}}$

From Table 4, it is known that

$$
\begin{aligned}
& M\left(S_{1}\right)=0.5167+0.2783 i_{1} \\
& M\left(S_{2}\right)=0.5275+0.2525 i_{2} \\
& M\left(S_{3}\right)=0.4938+0.1913 i_{3} \\
& M\left(S_{4}\right)=0.5708+0.0892 i_{4} .
\end{aligned}
$$


Table 9 Weighted comprehensive BCNs for each scheme

\begin{tabular}{ll}
\hline & $M\left(h_{k}\right)=A_{k}+B_{k} i_{k}$ \\
\hline$h_{1}$ & $M\left(h_{1}\right)=0.1787+0.0105 i_{1}$ \\
$h_{2}$ & $M\left(h_{2}\right)=0.2753+0.0559 i_{2}$ \\
$h_{3}$ & $M\left(h_{3}\right)=0.2560+0.0552 i_{3}$ \\
$h_{4}$ & $M\left(h_{4}\right)=0.2350+0.0351 i_{4}$ \\
\hline
\end{tabular}

According to Eq. (20),

$$
\begin{aligned}
& i_{1}=\frac{0.2783}{0.5167+0.2783}=0.3501, \\
& i_{2}=\frac{0.2525}{0.5275+0.2525}=0.3237, \\
& i_{3}=\frac{0.1913}{0.4938+0.1913}=0.2792, \\
& i_{4}=\frac{0.2892}{0.5708+0.0892}=0.1351 .
\end{aligned}
$$

Substituting these values in Eqs. (21-(24), respectively, we obtain

$M\left(S_{1}\right)=0.5167+0.2783 \times 0.3501=0.6141$,

$M\left(S_{2}\right)=0.5275+0.2525 \times 0.3237=0.6826$,

$M\left(S_{3}\right)=0.4938+0.1913 \times 0.2792=0.5471$,

$M\left(S_{4}\right)=0.5708+0.0892 \times 0.1351=0.5829$.

Because $M\left(S_{2}\right)>M\left(S_{1}\right)>M\left(S_{4}\right)>M\left(S_{3}\right)$, the preference order is $S_{2} \succ S_{1} \succ S_{4} \succ S_{3}$.

Example 4.2 (Gou and Xu 2016; Song et al. 2018) We evaluate four domestic hospitals in China to search for the one with the optimal resource allocation benefit. We consider three main criteria: the environmental factor of medical and health service $\left(c_{1}\right)$, personalized treatment optimization $\left(c_{2}\right)$, and social resource allocation and health services $\left(c_{3}\right)$. The corresponding weight vector is $w=(0.2,0.1,0.7)^{T}$. We consider the West China Hospital of Sichuan University $\left(h_{1}\right)$, Huashan Hospital of Fudan University $\left(h_{2}\right)$, Union Medical College Hospital $\left(h_{3}\right)$ and Chinese PLA General Hospital $\left(h_{4}\right)$. To overcome the information loss, invited experts give their evaluations with P-HFEs, which constitute the probabilistic hesitant fuzzy decision matrix $H=\left(h_{k t}\right)_{4 \times 3}$, as shown in Table 8.

We utilize the approach of Sect. 4.1.

Step 1. Calculate the weighted comprehensive BCNs for each scheme according to Eq. (19), and the results are listed in Table 9.

Step 2. Preliminarily rank the preference order of alternatives according to the $A_{k}$ values (probabilistic hesitant fuzzy centers) of the comprehensive BCNs in Table 9; sorting results are shown in Table 10.

From the results in Table 10, we can obtain that the ranking is $h_{2} \succ h_{3} \succ h_{4} \succ h_{1}$, and the optimal scheme is $h_{2}$, which is consistent with Gou and Xu (2016) and Song et al. (2018). However, if we consider the $i_{k}$ value of each alternative, the preference order of the four schemes may be different.
Step 3. Utilize $i_{k}$ in the comprehensive BCNs for hesitant fuzziness analysis.

We rank four alternatives when $i_{k}=-1,0,1$ in the weighted comprehensive evaluation BCN in Table 9, as shown in Table 11. From the hesitation itself, $i_{k}(k=$ $1,2,3,4)$ can take any value other than $-1,0,1$ in the interval $[-1,1]$.

Step 4. Give decision-making advice.

It can be seen from Table 11 that based on the synchronous value of $i_{k}$ in the comprehensive BCNs, the preference order of the four schemes is $h_{2} \succ h_{3} \succ h_{4} \succ h_{1}$. So, the optimal hospital is Huashan Hospital of Fudan University.

\subsection{Comparative analysis and conditional decision making}

We use Example 4.1 to further illustrate the objective significance of this work. We compare and analyze our work with Xia and Xu (Xia and Xu 2011). Xia and Xu rank the four schemes as $S_{4} \succ S_{2} \succ S_{1} \succ S_{3}$, which is consistent with the results in Table 5 , where $i_{k}=0$ in each hesitant fuzzy comprehensive BCN. In other words, it is a ranking result that does not consider the hesitant fuzzy characteristics, especially without the hesitant fuzzy intensity. Xia and Xu (2011) used a generalized hesitant fuzzy weighted geometric operator to make decisions. It is found that when $\lambda=1$ or $\lambda=2$, although the optimal alternative is still $S_{4}$, the ranking of the four alternatives becomes $S_{4} \succ S_{1} \succ S_{3} \succ S_{2}$. When $\lambda=5,10$ or 20 , the ranking becomes $S_{3} \succ S_{4} \succ S_{1} \succ S_{2}$; in this case, the optimal alternative is $S_{3}$. However, referring to Table 5, we can see that the ranking of the four alternatives is the result of avoiding the effect of hesitation and fuzziness of evaluation values on the ranking. The work of this paper shows that if we admit that the selection of the four alternatives is a hesitant fuzzy decision-making problem in a hesitant fuzzy environment, any of the four schemes can be optimal under a specific hesitant fuzzy comprehensive evaluation condition. Therefore, how to objectively determine some specific conditions in a given hesitant fuzzy environment to obtain the optimal scheme and the ranking of all schemes has a certain complexity, which comes from the superposition of hesitation and fuzziness. A systematic study and detailed analysis are needed to obtain a more objective conclusion. In this paper, by utilizing the systematicness, hesitant fuzziness, and determinability of the $\mathrm{BCN}$, the modulus of the $\mathrm{BCN}$ and the ratio formula of the hesitant fuzzy intensity function $i_{k}$ in the BCN are used to determine that the optimal scheme is $S_{2}$, and the ranking order is $S_{2} \succ S_{1} \succ S_{4} \succ S_{3}$. The common feature of these two approaches is based on the interaction between the hesitant fuzzy center and hesitant fuzzy intensity. The optimal scheme $S_{4}$ and ranking order $S_{4} \succ S_{2} \succ S_{1} \succ S_{3}$ obtained by Xia and Xu (2011) are only based on the hes- 
Table 10 Sorting of comprehensive evaluation BCNs in probabilistic hesitant fuzzy center $\left(i_{k}=0\right)$

\begin{tabular}{lllll}
\hline & $A_{k}\left(i_{k}=0\right)$ & Sort & $\begin{array}{l}\text { Sort using Guo and } \\
\text { Xu's method }\end{array}$ & $\begin{array}{l}\text { Compared with the } \\
\text { results in Gou and Xu } \\
(2016)\end{array}$ \\
\hline$h_{1}$ & 0.1787 & (4) & (4) & the same \\
$h_{2}$ & 0.2753 & (1) & (1) & the same \\
$h_{3}$ & 0.2560 & (2) & (2) & the same \\
$h_{4}$ & 0.2350 & (3) & (3) & the same \\
\hline
\end{tabular}

\begin{tabular}{lllllll}
\hline$M\left(h_{k}\right)$ & $i_{k}=-1$ & Ranking & $i_{k}=0$ & Ranking & $i_{k}=1$ & Ranking \\
\hline$M\left(h_{1}\right)$ & 0.1682 & $(4)$ & 0.1787 & (4) & 0.1891 & (4) \\
$M\left(h_{2}\right)$ & 0.2194 & $(1)$ & 0.2753 & $(1)$ & 0.3313 & (1) \\
$M\left(h_{3}\right)$ & 0.2008 & $(2)$ & 0.2560 & $(2)$ & 0.3112 & $(2)$ \\
$M\left(h_{4}\right)$ & 0.1999 & $(3)$ & 0.2350 & (3) & 0.2701 & (3) \\
\hline
\end{tabular}

Table 11 Ranking of each scheme when $i_{k}=-1,0,1$ with $\mathrm{P}$-HFE evaluation nection between hesitation and non-hesitation and the mutual conversion under certain conditions.

Some crucial advantages of the proposed decision model are:

1. It can truly reflect the hesitation psychology and performance of decision makers.

2. It studies hesitation and non-hesitation as a unity of opposites.

3. It provides a mathematical model for the mutual conversion of hesitation and non-hesitation, which is further ranked for the selection of a suitable object from a set of objects.

4. The proposed framework is also flexible to solve other multi-attribute decision making problems. To demonstrate the practical use, the selection problems of investment projects and those of domestic hospitals are presented.

Some of the limitations of the study are:

1. Theoretically, there are an infinite number of hesitation points in the model, which is in contradiction with limited decision-making. To solve this contradiction, we need to introduce new constraints.

2. The reasonable value of hesitant fuzzy intensity function and the strict algorithm rules for conditional decisionmaking need to be studied further.

In our future research, we will extend our proposed aggregation operators for handing multi-attribute decision-making problems in intuitionistic hesitant fuzzy, interval-valued intuitionistic hesitant fuzzy, and probabilistic interval-valued intuitionistic hesitant fuzzy; additionally, we will apply them to other multi-attribute decision-making problems. Although 
the $\mathrm{BCN}$ has been applied to other decision-making problems, this was its first application to HFMADM problems. The algorithm for the BCN decision making model, the reasonable value of hesitant fuzzy intensity function $i_{k}$, and the strict definition and algorithm rules of conditional decision making require further study.

Acknowledgements This work was supported in part by the National Natural Science Foundation of China (61802123, 61771193) and Primary Research and Development Plan of Zhejiang Province (2020C01097, 2020C01087).

\section{Declarations}

Conflict of interest The authors declare that they have no known competing financial interests or personal relationships that could have appeared to influence the work reported in this paper.

Ethical approval This article does not contain any studies with human participants or animals performed by any of the authors.

\section{References}

Ali A, Rashid T (2019) Hesitant fuzzy best-worst multi-criteria decision-making method and its applications. Int J Intell Syst 34(8):1953-1967

Cao YX, Zhou H, Wang JQ (2018) An approach to interval-valued intuitionistic stochastic multi-criteria decision-making using set pair analysis. Int J Mach Learn Cybern 9(4):629-640

Farhadinia B, Herrera-Viedma E (2019) Multiple criteria group decision making method based on extended hesitant fuzzy sets with unknown weight information. Appl Soft Comput 78:310-323

Garg H, Kumar K (2018) Distance measures for connection number sets based on set pair analysis and its applications to decision-making process. Appl Intelli 48(10):3346-3359

Grag H, Kumar K (2018) An advanced study on the similarity measures of intuitionistic fuzzy sets based on the set pair analysis theory and their application in decision making. Soft Comput 22:4959-4970

Garg H, Kumar K (2019) An advanced study on operations of connection number based on set pair analysis. Natl Acad Sci Lett India 42(4):351-354

Garg H, Kumar K (2020) A novel exponential distance and its based TOPSIS method for interval-valued intuitionistic fuzzy sets using connection number of SPA theory. Artif Intell Rev 53:595-624

Gou XJ, Xu ZS (2016) Novel basic operational laws for linguistic terms, hesitant fuzzy linguistic term sets ans probabilistic linguistic term sets. Inform Sci 372:407-427

He Y, Xu ZS (2019) Multi-attribute decision making methods based on reference ideal theory with probabilistc hesitant information. Exp Syst Appl 118:459-469

Kaplan RS, Norton DP (1996) The Balanced Scorecard: translating strategy into action. Harvard Business School Press, Boston

Krishankumar R, Ravichandran KS, Kar S, Gupta P, Mehlawat MK (2021) Double-hierarchy hesitant fuzzy linguistic term set-based decision framework for multi-attribute group decision-making. Soft Comput 25:2665-2685

Krishankumar R, Ravichandran KS, Gandomi AH, Kar S (2021) Interval-valued probabilistic hesitant fuzzy set-based framework for group decision-making with unknown weight information. Neural Comput Appl 33:2445-2457
Krishankumar R, Ravichandran KS, Liu P, Kar S, Gandomi AH (2021c) A decision framework under probabilisitc hesitant fuzzy environment with probability estimation for multi-criteria decision making. Neural Comput Appl. https://doi.org/10.1007/s00521020-05595-y

Kumar K, Garg H (2018) Connection number of set pair analysis based TOPSIS method on intuitionistic fuzzy sets and their application to decision making. Appl Intell 48:2112-2119

Kumar K, Garg H (2018) TOPSIS method based on the connection number of set pair analysis under interval-valued intuitionistic fuzzy set environment. Comput Appl Math 37:1319-1329

Liao HC, Wu XL, Liang XD, Xu JP, Herrera F (2018) A new hesitant fuzzy linguistic ORESTE method for hybrid multi-criteria decision making. IEEE Trans Fuzzy Syst 26(6):3793-3807

Ma ZZ, Zhu JJ, Ponnambalam K, Zhang ST (2019) A clustering method for large-scale group decision-making with mult-stage hesitant fuzzy linguistic terms. Inform Fusion 50:231-250

Mishra AR, Rani P, Krishankumar R, Ravichandran KS, Kar S (2021) An extended fuzzy decision-making framework using hesitant fuzzy sets for the drug selection to treat the mild symptoms of Coronavirus Disease 2019 (COVID-19). Appl Soft Comput 103:107155

Qin JD, Liu XW, Pedrycz W (2016) Frank aggregation operators and their application to hesitant fuzzy multiple attribute decision making. Appl Soft Comput 41:428-452

Rodriguez RM, Martinez L, Herrera F (2012) Hesitant fuzzy linguistic term sets for decision making. IEEE Trans Fuzzy Syst 20(1):109_ 119

Shen Q, Huang X, Liu Y, Jiang YL, Zhao KQ (2020) Multiattribute decision making based on the binary connection number in set pair analysis under an interval-valued intuitionistic fuzzy set environment. Soft Comput 24:7801-7809

Saha A, Dutta D, Kar S (2021) Some new hybird hesitant fuzzy weight aggregation operators based on Archimedean and Dombi operatons for multi-attribute decision making. Neural Comput Appl. https://doi.org/10.1007/s00521-020-05623-x

Song CY, Xu ZS, Zhao H (2018) A novel comparison of probabilistic hesitant fuzzy elements in multi-criteria decision making. Symmetry 10(5):177-188

Torra V, Narukawa Y (2009) On hesitant fuzzy sets and decision, In: 2009 IEEE international conference on fuzzy systems, pp 13781382

Torra V (2010) Hesitant fuzzy sets. Int J Intell Syst 25(6):529-539

Xia MM, Xu ZS (2011) Hesitant fuzzy information aggregation in decision making. Int J Approx Reason 52(3):395-407

Yang MS, Hussain Z (2019) Distance and similarity measures of hesitant fuzzy sets based on Hausdorff metric with applications to multi-criteria decision making and clustering. Soft Comput 23(14):5835-5848

Zhao H, Xu ZS, Wang H, Liu SS (2017) Hesitant fuzzy multi-attribute decision-making based on the minimum deviation method. Soft Comput 21(12):3439-3459

Zhu B (2014) Decision method for research and application based on preference relation. Southeast University, Nanjing, China

Zhao KQ (2008) The theoretical basis and basic algorithm of binary connection number $A+B i$ and its application in AI. CAAI Trans Intell Syst 5(5):454-457

Publisher's Note Springer Nature remains neutral with regard to jurisdictional claims in published maps and institutional affiliations. 